\title{
Delayed intra-tumoural haemorrhage in pineal germinoma: Case report and review ${ }^{*}$
}

\author{
Michael Colditz , Craig Winter \\ Kenneth G. Jamieson Department of Neurosurgery, Royal Brisbane \& Women’s Hospital, Herston, Australia \\ Email: \#michael.colditz@uqconnect.edu.au
}

Received 1 April 2013; revised 7 May 2013; accepted 20 June 2013

Copyright (C) 2013 Michael Colditz, Craig Winter. This is an open access article distributed under the Creative Commons Attribution License, which permits unrestricted use, distribution, and reproduction in any medium, provided the original work is properly cited.

\begin{abstract}
Intraparenchymal haemorrhage in a pineal germinoma is a very rare, though clinically significant event. We report the first case of a significantly delayed intraparenchymal haemorrhage in a pineal germinoma, 14 days after endoscopic third ventriculostomy (ETV), causing precipitous patient deterioration. We discuss potential contributing pathophysiological factors, and seek to illustrate that knowledge of its occurrence, and associated morbidity, is clinically significant in managing pineal germinoma patients with acute deterioration post obstructive hydrocephalus CSF diversion.
\end{abstract}

Keywords: Pineal Germinoma; Haemorrhage; Endoscopic Third Ventriculostomy (ETV); Tumour

\section{INTRODUCTION}

Pineal germinoma is a rare primary brain tumour (incidence $0.4 \%-3.4 \%[1]$ ) that classically presents with obstructive hydrocephalus or local mass effect [2]. Initial management involves treating the hydrocephalus with either endoscopic third ventriculostomy (ETV) or a shunt procedure, and gaining a tissue diagnosis to direct definitive treatment. Haemorrhage within pineal tumours is only rarely reported as a very early, but clinically significant, complication of treatment. We describe a very late and significant intraparenchymal haemorrhage with ventricular extension from a pineal germinoma, delayed at 14 days after ETV and biopsy.

\section{CASE REPORT}

A 15-year-old male presented with a four week history of progressive morning headache, nausea, vomiting, and

\footnotetext{
*No conflict of interest.

"Corresponding author.
}

diplopia.

Examination revealed paralysis of upward gaze, convergence-retraction nystagmus, and pseudo-Argyll-Robertson pupils consistent with Parinaud's dorsal midbrain syndrome, partial bilateral abducens nerve palsy, and papilloedema.

MRI brain demonstrated a large homogenous enhancing pineal tumour with prominent vascularity and associated obstructive triventricular hydrocephalus.

An ETV with tumour biopsy was performed; minor haemorrhage was noted intra operatively at the biopsy site which resolved quickly with irrigation. An extraventricular drain (EVD) was inserted as a precaution, and removed 24 hours later. The patient recovered well, with a significant improvement in the majority of his symptoms however the Parinaud's syndrome was slower to resolve. Histology, and elevated CSF beta-human chorionic gonadotropin and alpha fetoprotein levels, confirmed pineal germinoma.

Post-operative MR (Figure 1) confirmed patency of the third ventricle stoma, significant reduction in ventriculomegaly, with no intraventricular or intratumoural haemorrhage.

A CSF leak developed from the right frontal EVD site which resolved with simple re-suturing and $50 \mathrm{~mL}$ of CSF removed via lumbar puncture. Given the patency of the stoma it was considered possible that an element of communicating hydrocephalus may have developed. The patient remained clinically very well, until a sudden drop in conscious level to GCS 8 on day 14, accompanied by urinary incontinence and emesis. The patient was intubated and emergent CT imaging (Figure 2) was performed, which revealed a large diffuse parenchymal haemorrhage within the tumour, intra-ventricular haematoma and acute hydrocephalus.

A right frontal EVD was reinserted immediately. PostEVD CT imaging revealed a dilatation of the left ventricle secondary to castes of blood situated both at the fo- 


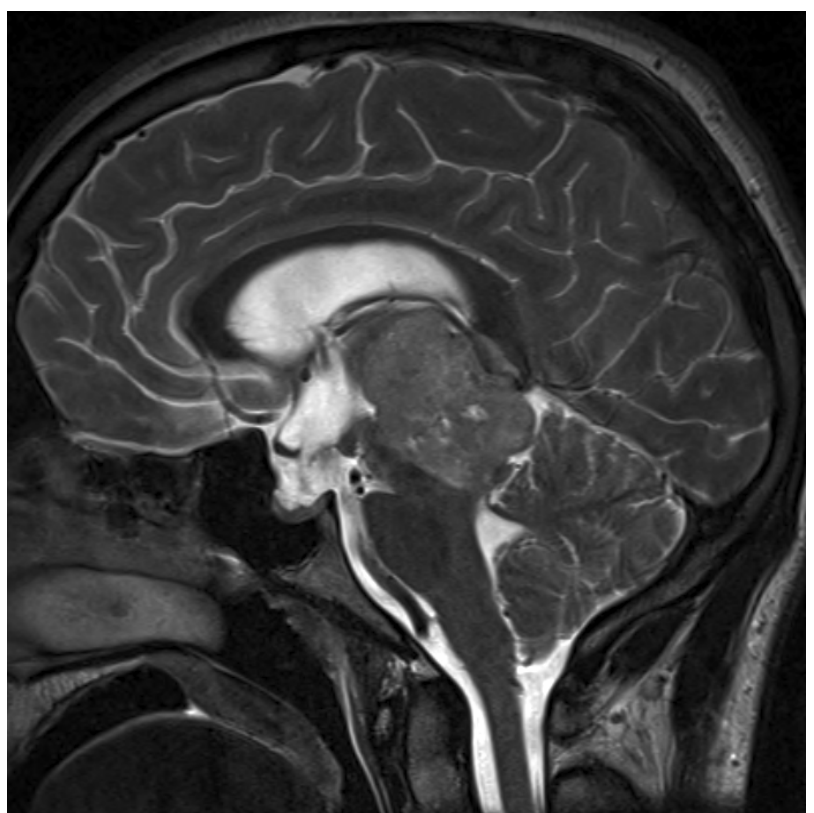

Figure 1. T2 sagittal MRI brain: Two days post ETV. Third ventricle stoma patent.

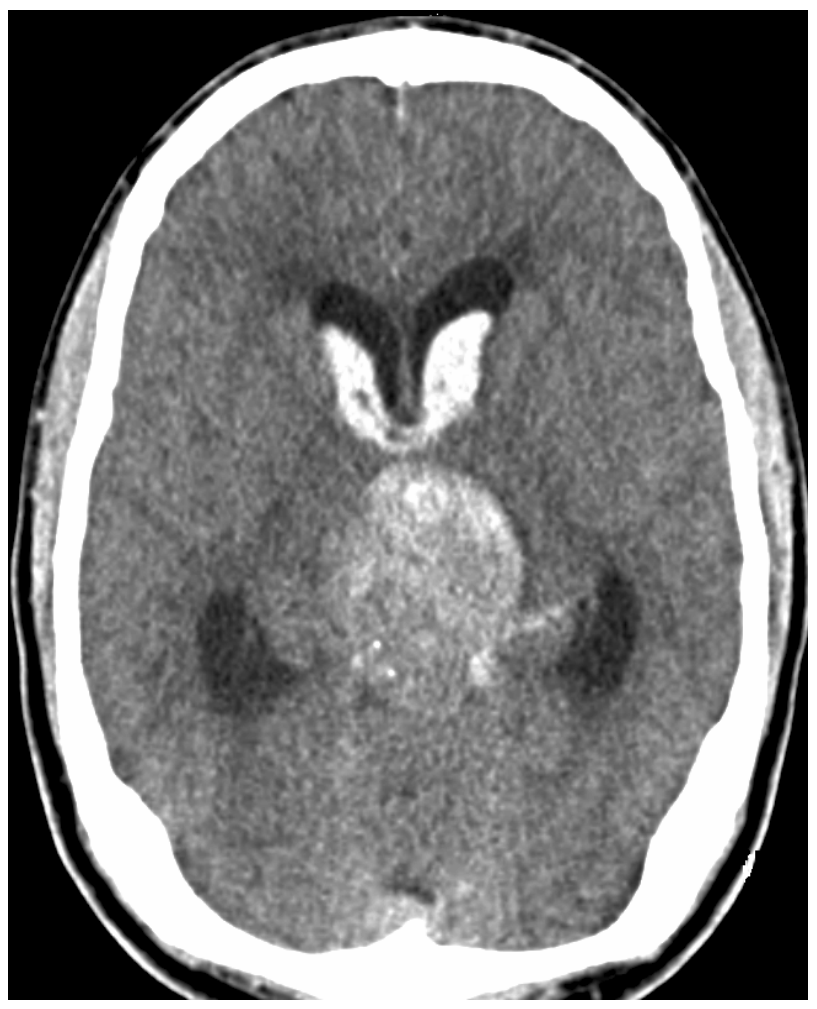

Figure 2. CT contrast brain: Diffuse enlargement of pineal tumour secondary to large intraparenchymal haemorrhage with intraventricular extension and acute hydrocephalus.

ramina of Monroe and overlying the third ventricle stoma. This prompted placement of a left frontal EVD.

The EVDs were gradually weaned and chemotherapy commenced to reduce the tumour bulk. The patient was discharged home a fortnight later with considerable reduction of symptoms, and serial imaging confirming progressive reduction in tumour size.

\section{DISCUSSION}

Intra-tumoural haemorrhage in pineal germinomas is a rarely reported complication following treatment of obstructive hydrocephalus by either ETV or shunt procedure. Large clinical studies assessing ETV complications do not describe this specific type of post ETV haemorrhage [3-7]. It has however been reported, only in the acute setting, in a small number of individual case reports following CSF diversion for pineal tumours without biopsy. The presentations varied from a small asymptomatic bleed [8], acute deterioration at two hours [9] and on day two [10], and a fatal bleed at 5 hours [11]. Clinically significant haemorrhage post-CSF diversion for obstructive hydrocephalus caused by other posterior fossa tumours, again without biopsy, has also been described [12-19].

It has been hypothesised that altered CSF dynamics post CSF diversion is the major precipitating event causing tumour haemorrhage [9]. Proposed pathophysiological mechanisms implicate the acute reduction of CSF pressure in one ventricular compartment resulting in an increased transmural pressure gradient across the tumour, with progressive distortion of fragile tumour vessels. Resultant tumour movement may further compress venous drainage leading to venous hypertension, increased tumour oedema and spontaneous parenchymal haemorrhage $[12,20]$. All clinically relevant tumour haemorrhages reported have occurred by Day 2, which is consistent with the proposed mechanism of haemorrhage secondary to acute changes in intracranial pressure (ICP). The only other reported case of pineal haemorrhage was a clinically insignificant bleed at Day 10, only incidentally noted on preoperative imaging [8].

The aetiology for such a delayed and clinically significant tumour haemorrhage in our patient at Day 14 is unclear. Although minor haemorrhage followed the endoscopic biopsy (a well-recognised phenomenon), postoperative imaging confirmed that this was not significant. We do not feel that the diffuse nature of the haemorrhage in our patient related to either the biopsy or to the acute changes in ICP responsible for the reported clinically significant haemorrhages.

The possible development of an element of communicating hydrocephalus (albeit mild) may have resulted from the initial biopsy haemorrhage. Persistently elevated ICP may have contributed by exacerbating venous congestion, leading to tumoural microhaemorrhage, oedema, venous infarction and final large haemorrhage [21].

Pineal germinomas occasionally present with a spon- 
taneous bleed causing subarachnoid haemorrhage [21-24]. Particular tumour features associated with spontaneous haemorrhage [24] may have predisposed this prominently vascular, and untreated, tumour to haemorrhage independent of ETV effect.

Whether the cause of the haemorrhage is related to altered CSF dynamics, transmural mechanical stress, vascular congestion, elevated ICP, or simply stochastic, remains equivocal. It is, however, a previously undescribed phenomenon, that is a clinically important differential for the patient with delayed-acute deterioration.

\section{CONCLUSION}

Pineal germinoma haemorrhage post ETV is a rare and clinically relevant complication, previously described as occurring only in the acute setting. We report the first case of a haemorrhage occurring in a very delayed fashion (Day 14) with resultant intraventricular haemorrhage, and hydrocephalus (despite ETV) that caused significant acute patient deterioration. This significant complication has associated morbidity, and knowledge of its occurrence deserves consideration and action from the surgeon managing patients after CSF diversion for obstructive hydrocephalus in the setting of a pineal germinoma.

\section{REFERENCES}

[1] Srinivasan, N., Pakala, A., Mukkamalla, C. and Oswal, A. (2010) Pineal germinoma. Southern Medical Journal, 103, 1031-1037. doi:10.1097/SMJ.0b013e3181ebeeff

[2] Clark, A., Sughrue, M.E., Ivan, M., Aranda, D., Rutkowski, M., Kane, A., Chang, S. and Parsa, A. (2010) Factors influencing overall survival rates for patients with pineocytoma. Journal of Neuro-Oncology, 100, 255-260. doi:10.1007/s11060-010-0189-6

[3] Bouras, T. and Sgouros, S. (2011) Complications of endoscopic third ventriculostomy. Journal of Neurosurgery: Pediatrics, 7, 643-649. doi:10.3171/2011.4.PEDS10503

[4] Cipri, S., Gangemi, A., Cafarelli, F., Messina, G., Iacopino, P., Al Sayyad, S., Capua, A., Comi, M. and Musitano, A. (2005) Neuroendoscopic management of hydrocephalus secondary to midline and pineal lesions. Journal of Neurosurgical Sciences, 49, 97-106.

[5] O’Brien, D.F., Hayhurst, C., Pizer, B. and Mallucci, C.L. (2005) Outcomes in patients undergoing single-trajectory endoscopic third ventriculostomy and endoscopic biopsy for midline tumors presenting with obstructive hydrocephalus. Journal of Neurosurgery, 105, 219-226.

[6] Sacko, O., Boetto, S., Lauwers-Cances, V., Dupuy, M. and Roux, F.E. (2010) Endoscopic third ventriculostomy: Outcome analysis in 368 procedures. Journal of Neurosurgery: Pediatrics, 5, 68-74. doi:10.3171/2009.8.PEDS08108

[7] Hayashi, N., Murai, H., Ishihara, S., Kitamura, T., Miki, T., Miwa, T., Miyajima, M., Nishiyama, K., Ohira, T., Ono, S., Suzuki, T., Takano, S., Date, I., Saeki, N. and
Endo, S. (2011) Nationwide investigation of the current status of therapeutic neuroendoscopy for ventricular and paraventricular tumors in Japan. Journal of Neurosurgery, 115, 1147-1157. doi:10.3171/2011.7.JNS101976

[8] Harada, K., Hayashi, T., Anegawa, S., Torigoe, R., Maeda, K., Toda, K., Sugita, Y. and Utsunomiya, H. (1993) Pineocytoma with intratumoral hemorrhage following ventriculoperitoneal shunt-Case report. Neurologia Medico-Chirurgica (Tokyo), 33, 836-838.

doi:10.2176/nmc.33.836

[9] Wong, T.T., Yen, S.H., Ho, D.M., Chang, F.C. and Chang, K.P. (2003) Pineal germinoma with intratumoral hemorrhage after neuroendoscopic tumor biopsy. Child's Nervous System, 19, 769-772. doi:10.1007/s00381-003-0800-7

[10] Matsumoto, K., Imaoka, T., Tomita, S. and Ohmoto, T. (1997) Pineocytoma with massic intratumoral hemorrhage after ventriculoperitoneal shunt. Neurologia Medico-Chirurgica (Tokyo), 37, 911-915. doi:10.2176/nmc.37.911

[11] Waga, S., Shimizu, T., Shimosaka, S. and Tochio, H. (1981) Intratumoral hemorrhage after a ventriculoperitoneal shunting procedure. Neurosurgery, 9, 249-252. doi:10.1227/00006123-198109000-00005

[12] Salunke, P., Sharma, M. and Bhardwaj, L. (2011) Bleed and Herniation in tumors compressing the ventricular system: perils of preoperative CSF diversion. Journal of Neurosurgical Anesthesiology, 23, 268-270. doi:10.1097/ANA.0b013e31821b9d95

[13] Murata, T., Shigeta, H., Sato, A., Tanaka, Y. and Hongo, K. (2006) Intratumoral hemorrhage in choriocarcinoma during neuroendoscopic third ventriculostomy: Case report. No Shinkei Geka. Neurological Surgery, 4, 305-310.

[14] Vaquero, J., Cabezudo, J.M., De Solo, R.G. and Nombela, L. (1981) Intratumoral hemorrhage in posterior fossa tumors after ventricular drainage: Report of two cases. Journal of Neurosurgery, 54, 406-408. doi:10.3171/jns.1981.54.3.0406

[15] Santhanam, R., Balasubramaniam, A. and Chandramouli, B.A. (2009) Fatal intratumoral hemorrhage in posterior fossa tumors following ventriculoperitoneal shunt. Journal of Clinical Neuroscience, 16, 135-137. doi:10.1016/j.jocn.2008.02.016

[16] Kasliwal, M.K., Agrawal, D. and Sharma, B.S. (2008) Fatal intratumoral hemorrhage following ventriculo-peritoneal shunt. Turkish Neurosurgery, 18, 436-438.

[17] Schroeder, H.W., Niendorf, W.R., Schwesinger, G. and Gaab, M.R. (1995) Fatal tumor hemorrhage after ventriculoperitoneal shunting. Case report. Neuropediatrics, 26, 177-179. doi:10.1055/s-2007-979750

[18] Zuccarello, M., Dollo, C. and Carollo, C. (1985) Spontaneous intratumoral hemorrhage after ventriculoperitoneal shunting. Neurosurgery, 16, 245-246. doi:10.1227/00006123-198502000-00024

[19] Epstein, F. and Murali, R. (1978) Pediatric posterior fossa tumors: Hazards of the "preoperative" shunt. Neurosurgery, 3, 348-350. doi:10.1227/00006123-197811000-00003 
[20] Singha, S.K., Chatterjee, N. and Neema, P.K. (2009) Reverse herniation of brain: A less recognized complication in a patient with midline posterior fossa tumor postendoscopic third ventriculostomy. Journal of Neurosurgical Anesthesiology, 21, 354-355.

doi:10.1097/ANA.0b013e3181ac8edb

[21] Hung, Y.C., Lee, E.J., Wang, L.C., Chen, H.H., Yan, J.J. and Yu, C.Y. (1999) Mixed germ cell tumor presenting as intratumoral hemorrhage: Report of a case originated from the pineal region. The Kaohsiung Journal of Medical Sciences, 15, 498-503.

[22] Hata, N., Inamura, T., Matsushima, T., Yoshimoto, K.,
Ikezaki, K., Nakamizo, A., Inoha, S. and Fukui, M. (2002) A case of pineoblastoma primary presenting a pineal hemorrhage causing obstructive hydrocephalus. No Shinkei Geka. Neurological Surgery, 30, 65-70.

[23] Steinbok, P., Dolman, C.L. and Kaan, K. (1977) Pineocytomas presenting as subarachnoid hemorrhage: Report of two cases. Journal of Neurosurgery, 47, 776-780.

[24] Kondziolka, D., Bernstein, M., Resch, L., Tator, C.H., Fleming, J.F.R., Vanderlinden, R.G. and Schutz, H. (1987) Significance of hemorrhage into brain tumors: Clinicopathological study. Journal of Neurosurgery, 67, 852-857. doi:10.3171/jns.1987.67.6.0852 